\title{
Adjoint-based shape optimization of fin geometry for heat transfer enhancement in solidification problem
}

\author{
Kenichi MORIMOTO*, Hidenori KINOSHITA* and Yuji SUZUKI* \\ * Department of Mechanical Engineering, The University of Tokyo \\ 7-3-1 Hongo, Bunkyo-ku Tokyo 113-8656, Japan \\ E-mail: morimoto@mesl.t.u-tokyo.ac.jp
}

Received 6 July 2016

\begin{abstract}
In the present study, an adjoint-based shape-optimization method is formulated for heat transfer enhancement in liquid-solid phase change problems, in which heat conduction is dominant. In the present shape-optimization scheme, extended heat transfer surfaces with constant wall temperature are allowed to deform based on variational information of a cost functional, which is obtained from the physical temperature and adjoint enthalpy fields. In the computation of the developed scheme with an enthalpy-based formulation, a meshless local Petrov-Galerkin (MLPG) method is implemented for dealing with the complex boundary shape. For high-resolution analyses, a bubble-mesh method for boundary-fitted node arrangement in a well-controlled manner is employed, combined with a high-efficiency searching algorithm for choosing the neighboring bubbles interacted with each other. In the shape evolution process for different initial fin shapes, it is demonstrated that, within a certain range of the initial state, the present shape-optimization scheme leads to tree-like fin shapes that achieve the temperature field with global similarity.
\end{abstract}

Key words : Shape optimization, Adjoint analysis, Solidification, Meshless analysis

\section{Introduction}

Recently, the optimal design methodology for various heat transfer-related equipment, in which heat and fluid phenomena play an important role in the overall performance, has gained renewed attention. In the adjoint-based optimization approach, a set of adjoint variables are introduced to obtain the derivative information of a cost function with respect to the variation of the control inputs. In thermo-fluids systems governed by nonlinear partial differential equations (PDEs), the adjoint equations correspond to Riccati equations in systems governed by ordinary differential equations. The adjoint-based approach has advantages over stochastic approach, such as genetic algorithms (Goldberg, 1989), in its applicability to arbitrary forms of the control input that cannot be parameterized with known base functions. At a relatively-small computational cost, the adjoint-based analysis ensures local optimality (Pirroneau, 1984) around a possible solution, and even provides local sensitivity information of the control input to the PDE system. With such unique features, the adjoint-based optimization approach is expected to serve as one of the key ingredients in the optimal design of high-performance thermo-fluids devices/systems.

Over the past several decades, remarkable advances have been made in the adjoint-based optimization in heat and fluid flow problems. In the framework of inverse heat transfer problems, the adjoint variable methods have been established for various applications (Alifanov, 1974; Subaşi, 2002; Loulou \& Scott, 2006). In fluid mechanics fields, since the pioneering study on shape design in Stokes flow problem (Pironneau, 1973), further extensions to laminar and turbulent flow problems (Çabuk \& Modi, 1992; Lim \& Choi, 2004) have been accomplished owing partially to the increase of computational power. In aerodynamics application, after the work on inviscid Euler equation system (Jameson, 1988), much work has been done, and even the wing design for full-scale transonic jet has been reported (Reuther et al., 1996; Mohammadi \& Pironneau, 2004). Very recently, further applications to high Reynolds number flow have been tackled (Zymaris et al., 2010; Walther \& Nadarajah, 2012; Papoutsis-Kiachagias \& Giannakoglou, 2016). The applications of optimal control theory to turbulence control problems (Bewley et al., 2001; Kasagi et al., 
2012) have also brought about great success. Other applications of the adjoint-based designing approach have been also reported (Molho et al., 2001).

In the field of structural mechanics, topology optimization methods have been intensively studied in recent years (e.g., Bendsøe \& Sigmund, 2004; Allaire et al., 2004). More recently, the topology optimization techniques have been also extended to various problems with heat conduction (Gersborg-Hansen et al., 2006; Zhuang et al., 2007; Iga et al., 2009) and Navier-Stokes flows (Zhou \& Li, 2008; Deng et al., 2011). In shape optimization methods, the geometrical shape of the boundary surface, which is represented by geometrical parameters, is to be optimized. On the other hand, in topology optimization methods, the entire structure including the boundary surface can change its topology during the optimization (Reynolds et al., 1999). In homogenization approach (Bendsøe \& Sigmund, 2004), the boundary shape is obtained by a grey-scale image, representing contours of material properties such as the porosity or the density of the elements. In level-set approach (Allaire et al., 2004), the level-set function (Sethian, 1999) is employed to describe the design boundary. The immersed boundary method (Peskin, 1977; Mittal \& Iaccarino, 2005) can also be incorporated to obtain the physical fields (Sethian \& Wiegmann, 2000). However, in the optimization of the boundary shape in heat transfer and fluid flow problems with forced convection involving high Reynolds number turbulent flow and/or conjugate heat transfer involving different materials with different properties, accurate prediction of the wall heat flux and the wall shear stress is of critical importance. Therefore, the straightforward shape-representation approach, with which the boundary shape is physically determined by a set of nodal points, is employed in the present study.

We previously demonstrated the effectiveness of the adjoint-based analysis on the optimization of convective heat transfer problem in designing laminar-flow compact heat exchangers (Morimoto et al., 2010). The momentum and heat transfer characteristics were simultaneously considered, and the shape optimization of the heat transfer surface geometry for maximizing the $j / f$ factor has been established for the first time. Also, we have applied the adjoint-based analysis to the heat conduction control of semiconductor pulsed lasers for reducing the temperature variation inside the active region (Morimoto \& Suzuki, 2014). The present study aims to establish an adjoint-based shape-optimization method for heat transfer processes involving solidification phenomena. Possible targets of the present shape optimization method include, for example, thermal storage and cooling techniques using phase change materials (Sharma et al., 2009; Kandasamy et al., 2008). For phase-change heat conduction problems, the adjoint analysis has been previously applied to boundary heat flux control (Zabaras \& Kang, 1993), but to the authors' best knowledge no work has been reported in the context of the shape optimization.

In the present study, we develop a shape-optimization method of fin geometry for heat transfer enhancement in liquid/solid phase-change problem. As our first step, only heat conduction and phase change are considered, and convection is neglected. We formulate an adjoint-based shape-optimization scheme and verify its effectiveness based on a series of high-resolution meshless computation. We employ bubble-mesh method for boundary-fitted node arrangement in the meshless analysis, combined with a high-efficiency node-searching algorithm. We show the effect of the initial fin geometry upon the shape evolution process during the present shape optimization.

\section{Formulation of adjoint analysis}

Figure 1 shows the schematic of the present shape optimization. The domain $\Omega$ is filled with liquid, of which the temperature is above its freezing point. The liquid is cooled by the lower boundary surface $\Gamma_{\mathrm{D}}$ and the fin $\Gamma_{\mathrm{M}}$. The shape of the fin geometry $\Gamma_{\mathrm{M}}$ is to be optimized for enhanced solidification process under the isothermal boundary condition. The adiabatic boundaries on the sides and the top are denoted by $\Gamma_{\mathrm{N}}$ (dashed lines).

In the present heat transfer analyses, the enthalpy method (Swaminathan \& Voller, 1993) is adopted for simulating the motion of the phase-change interface. In the enthalpy method using the apparent heat capacity formulation (Thakur et al., 2011), the governing equation of the heat conduction involving phase change is written as:

$$
\frac{\mathrm{d} \hat{H}}{\mathrm{~d} \hat{T}} \frac{\partial \hat{T}}{\partial \hat{t}}=\nabla \cdot(\hat{k} \nabla \hat{T})
$$

where $T$ and $H$ are the temperature and the enthalpy variables, respectively. We assume a linearized temperature-enthalpy relation using a small-width mushy zone (Voller, 1996). The enthalpy is expressed as a function of the temperature as follows: 


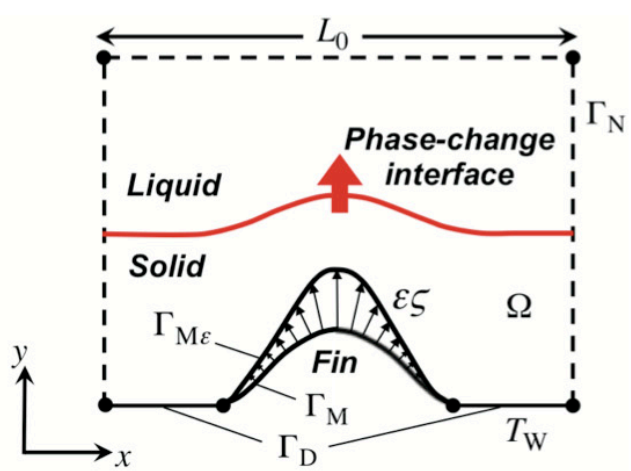

Fig. 1 Schematic of the present shape optimization for enhancement of solidification process.

$$
\hat{H}=\left\{\begin{array}{l}
\hat{T} \text { for } \hat{T}<-\hat{\varepsilon}_{\mathrm{T}}, \\
1 / 2 \cdot\left(1+c_{\mathrm{L}} / c_{\mathrm{S}}+1 /\left(\varepsilon_{\mathrm{T}} S t e\right)\right) \hat{T}+1 / 2 \cdot\left(-1+c_{\mathrm{L}} / c_{\mathrm{S}}+1 /\left(\varepsilon_{\mathrm{T}} S t e\right)\right) \hat{\varepsilon}_{\mathrm{T}} \text { for }-\hat{\varepsilon}_{\mathrm{T}} \leq \hat{T} \leq \hat{\varepsilon}_{\mathrm{T}}, \\
1 / \text { Ste }+\left(c_{\mathrm{L}} / c_{\mathrm{S}}\right) \hat{T} \text { for } \hat{\varepsilon}_{\mathrm{T}}<\hat{T} .
\end{array}\right.
$$

Here, each quantity is non-dimensionalized as follows:

$$
\begin{aligned}
& \hat{T}=\left(T-T_{\mathrm{M}}\right) /\left(T_{\mathrm{M}}-T_{\mathrm{W}}\right), \hat{H}=\left(H-c_{\mathrm{S}} T_{\mathrm{M}}\right) / c_{\mathrm{S}}\left(T_{\mathrm{M}}-T_{\mathrm{W}}\right), \\
& \hat{x}=x / L_{0}, \hat{y}=y / L_{0}, \hat{t}=t k_{\mathrm{S}} /\left(\rho c_{\mathrm{S}} L_{0}^{2}\right), \hat{k}=k / k_{\mathrm{S}}, \hat{\varepsilon}_{T}=\varepsilon_{T} /\left(T_{\mathrm{M}}-T_{\mathrm{W}}\right) .
\end{aligned}
$$

with the characteristic length $L_{0}$ (the width of the whole computational domain), the freezing temperature $T_{\mathrm{M}}$, the constant wall temperature $T_{\mathrm{W}}$, the density $\rho$, the heat capacity $c$, the thermal conductivity $k$, the latent heat $L$, and the small width of temperature, $\varepsilon_{\mathrm{T}}$. The subscripts $\mathrm{S}$ and $\mathrm{L}$ denote the value of the solid and the liquid, respectively. The Stefan number is defined by Ste $=c_{\mathrm{S}}\left(T_{\mathrm{M}}-T_{\mathrm{W}}\right) / L$. Hereafter, the symbol for dimensionless variables $\left({ }^{\wedge}\right)$ is omitted.

In the present adjoint analysis, the cost functional to be maximized is defined as:

$$
J=\left.\int_{\Omega}\left(H-H_{0}\right)^{2}\right|_{t=t_{\max }} \mathrm{d} \Omega,
$$

where the subscript 0 denotes the initial value at $t=0$. The present cost functional corresponds to the integral of the square of the change of the enthalpy field over the whole domain. The present shape optimization aims to maximize the amount of heat transfer in a prescribed time duration $t_{\max }$. With larger fins, heat conduction to/from the liquid is enhanced at the cost of reducing the liquid volume, which corresponds to smaller maximum heat that can be transferred to/from the liquid.

In the present optimization, the shape of the boundary $\Gamma_{\mathrm{M}}$ is modified in an iterative way based on the spatial distribution of the surface deformation $\zeta\left(\Gamma_{\mathrm{M}}\right)$, which is derived from the adjoint-based heat conduction analysis. The surface deformation in the wall-normal direction, $\zeta$, is obtained at each point on the fin surface to be modified. By correlating the perturbation field of the physical enthalpy to the adjoint enthaply field $H^{*}$, the first variation of the cost functional with respect to the surface deformation, which corresponds to the Fréchet derivative of $J$ (e.g., Lions, 1971), can be derived as:

$$
\begin{aligned}
\delta J & \equiv\left(J\left(\Gamma_{\mathrm{M} \varepsilon}\right)-J\left(\Gamma_{\mathrm{M}}\right)\right) / \varepsilon \\
& =\left.\int_{\Omega} 2 \frac{\mathrm{d} H}{\mathrm{~d} T} T^{\prime}\left(H-H_{0}\right)\right|_{t=t_{\max }} \mathrm{d} \Omega+\left.\int_{\Gamma_{\mathrm{M}}} \zeta\left(1+H_{0}\right)^{2}\right|_{t=t_{\max }} \mathrm{d} \Omega+\int_{0}^{t_{\max }} \int_{\Omega} H^{*}\left\{\frac{\mathrm{d} H}{\mathrm{~d} T} \frac{\partial T^{\prime}}{\partial t}-\frac{\partial}{\partial x}\left(k \frac{\partial T^{\prime}}{\partial x}\right)-\frac{\partial}{\partial y}\left(k \frac{\partial T^{\prime}}{\partial y}\right)\right\} \mathrm{d} \Omega \mathrm{d} t \\
& =\left.\int_{\Omega} 2 \frac{\mathrm{d} H}{\mathrm{~d} T} T^{\prime}\left(H-H_{0}\right)\right|_{t=t_{\max }} \mathrm{d} \Omega-\int_{0}^{t_{\max }} \int_{\Omega} T^{\prime}\left\{\frac{\mathrm{d} H}{\mathrm{~d} T} \frac{\partial H^{*}}{\partial t}+\frac{\partial}{\partial x}\left(k \frac{\partial H^{*}}{\partial x}\right)+\frac{\partial}{\partial y}\left(k \frac{\partial H^{*}}{\partial y}\right)\right\} \mathrm{d} \Omega \mathrm{d} t \\
& +\left.\int_{\Gamma_{\mathrm{M}}} \zeta\left(1+H_{0}\right)^{2}\right|_{t=t_{\max }} \mathrm{d} \Omega+\int_{\Omega}\left[\frac{\mathrm{d} H}{\mathrm{~d} T} T^{\prime} H^{*}\right]_{0}^{t_{\max }} \mathrm{d} \Omega+\int_{0}^{t_{\max }} \int_{\Gamma_{\mathrm{M}}} T^{\prime}\left(k \frac{\partial H^{*}}{\partial n}\right) \mathrm{d} \Gamma \mathrm{d} t-\int_{0}^{t_{\max }} \int_{\Gamma_{\mathrm{M}}} H^{*}\left(k \frac{\partial T^{\prime}}{\partial n}\right) \mathrm{d} \Gamma \mathrm{d} t,
\end{aligned}
$$


where $T^{\prime}$ represents the perturbed temperature field, and the divergence theorem is applied in the derivation. The second term in the R.H.S. of the second line of Eq. (5) arises from the change of the integral domain. The perturbation variables satisfy the following equation:

$$
\frac{\partial H^{\prime}}{\partial t}=\frac{\partial}{\partial x}\left(k \frac{\partial T^{\prime}}{\partial x}\right)+\frac{\partial}{\partial y}\left(k \frac{\partial T^{\prime}}{\partial y}\right) \text { in } \Omega,
$$

with the initial and boundary conditions:

$$
\left\{\begin{array}{l}
\left.T^{\prime}\right|_{t=0}=\left.H^{\prime}\right|_{t=0}=0 \\
\partial T^{\prime} / \partial n=0 \text { on } \Gamma_{\mathrm{N}} .
\end{array}\right.
$$

On the isothermal boundary, the perturbation variable is related to its physical variable as follows:

$$
T^{\prime}=-\zeta \cdot \partial T / \partial n, H^{\prime}=-\zeta \cdot \partial H / \partial n \text { on } \Gamma_{\mathrm{D}} \text { and } \Gamma_{\mathrm{M}}
$$

which can be deduced from the Taylor series expansions of $T$ and $H$ with respect to the small magnitude of the surface deformation $\varepsilon$.

Based on Eqs. (5)-(8), the adjoint equation can be derived as:

$$
\frac{\mathrm{d} H}{\mathrm{~d} T} \frac{\partial H^{*}}{\partial t}+\frac{\partial}{\partial x}\left(k \frac{\partial H^{*}}{\partial x}\right)+\frac{\partial}{\partial y}\left(k \frac{\partial H^{*}}{\partial y}\right)=0,
$$

with the following initial and boundary conditions:

$$
\left\{\begin{array}{l}
\left.H^{*}\right|_{t=t_{\max }}=-\left.2\left(H-H_{0}\right)\right|_{t=t_{\max }} \\
H^{*}=0 \text { on } \Gamma_{\mathrm{D}} \text { and } \Gamma_{\mathrm{M}}, \\
\frac{\partial H^{*}}{\partial n}=0 \text { on } \Gamma_{\mathrm{N}} .
\end{array}\right.
$$

It is noted here that, since the time advancement of the adjoint field is in the opposite direction to the physical field, the initial condition for the adjoint field is specified at $t=t_{\max }$.

The surface shape is updated with a gradient-based method, while ensuring the monotonic change of the cost functional. Since $\delta J$ expressed in Eq. (5) can be rearranged to

$$
\delta J=\int_{\Gamma_{\mathrm{M}}}\left\{-\left(1+H_{0}\right)^{2}+\int_{0}^{t_{\max }} k \frac{\partial T}{\partial n} \frac{\partial H^{*}}{\partial n} d t\right\} \zeta \mathrm{d} \Gamma,
$$

the shape deformation rule can be finally obtained as:

$$
\zeta=-\left(1+H_{0}\right)^{2}+\int_{0}^{t_{\max }} k \frac{\partial T}{\partial n} \frac{\partial H^{*}}{\partial n} \mathrm{~d} t,
$$

which ensures $\delta J \geq 0$ during the shape optimization. The first term of the R.H.S. of Eq. (12) means the penalty of the increase in the fin volume, and the second term represents the sensitivity information of the wall heat flux and its adjoint field.

In the present optimization, for ease of its implementation, a steepest descent algorithm is chosen. Other gradient methods such as the conjugate gradient method can also be used without much difficulty. During the present computation, the step size parameter $\varepsilon$ is not necessarily the optimal value. As explained later, the value of $\varepsilon$ is locally adjusted in the update process of the boundary nodes.

\section{Numerical methods}

\subsection{MLPG method}

In the present computation, we employ a meshless local Petrov-Galerkin (MLPG) method (Atluri \& Zhu, 1998) that is considered to be suited for dealing with complex boundary geometry. In the MLPG method, a weak form of the 
governing equation is discretized in space and time using nodes scattered over the computational domain. In the present MLPG analysis, the spatial interpolation is based on a moving least square (MLS) approximation (Liu \& Gu, 2005). The test function in the weak form of the governing equation and the weight function for the MLS approximation are given by the same fourth-order spline function. For the base function of the MLS interpolation, the second-order polynomial is used. The support domain around each node is set as the circular domain with its influential radius $\alpha_{S} R$. Here, $R$ denotes a representative node spacing, and $\alpha_{S}$ is a size factor. The local quadrature domain is formed by circular region around each node with its radius $\alpha_{\mathrm{G}} R$., and the Gauss quadrature rule (Zienkiewicz et al., 2005) is applied. In the present computation, constant values of $\alpha_{S}=2.6$ and $\alpha_{G}=0.5$ are employed. For the time advancement, a predictor-corrector method (Lewis \& Roberts, 1987) is employed for treating the nonlinearity of the present calculation.

\subsection{Bubble mesh method}

For high-resolution computation, nodes for the present MLPG analysis are generated by a node arrangement technique based on bubble mesh method (Shimada \& Gossard, 1998) that was originally developed to make triangular meshes for finite element simulation. Here we follow the bubble mesh scheme in order to arrange boundary-fitted nodes while keeping the node spacing uniform for different boundary shapes. The bubble generation scheme consists of the following three procedures:

(i) Placement of the initial bubbles,

(ii) Relaxation of the bubble-bubble interaction force,

(iii) Adjustment of the packing factor around each bubble.

In Procedure (ii), the bubbles, which are assumed to be spheres with given radii, are connected through springs and dampers. The stable distance between two bubbles is defined as $r_{0}=d_{i}+d_{j}$, where $d_{i}$ denotes the radius of the $i$-th bubble. The spring has stiffness dependent on the distance between two bubbles, with $k_{0}$ being the spring constant at the stable distance. The force is defined as a cubic function of the distance between the bubbles, $r$, which is similar to that in Shimada \& Gossard (1998):

$$
\begin{aligned}
& f(r)=\left\{\begin{array}{l}
c_{3} r^{3}+c_{2} r^{2}+c_{1} r+c_{0} \text { for } 0 \leq r \leq \alpha_{B} r_{0}, \\
0 \text { for } r<0, \alpha_{B} r_{0}<r,
\end{array}\right. \\
& f\left(r_{0}\right)=0, f\left(\alpha_{B} r_{0}\right)=0, f^{\prime}(0)=0, f^{\prime}\left(r_{0}\right)=-k_{0} .
\end{aligned}
$$

In the present optimization, the bubble mesh is generated after each iteration step of the fin shape modification. The following assumptions/constraints are imposed on the nodal displacement:

1) The nodes are distributed equally along the arc-length direction of the fin surface $\left(\Gamma_{M}\right)$. Here, the boundary $\Gamma_{M}$ is assumed as a set of line segments connecting the two neighboring nodes.

2) Nodes on the four corners of the domain $\Omega$ and nodes at both ends of $\Gamma_{M}$ are fixed. The nodes on the boundary edges are also fixed in the two-dimensional bubble generation inside the domain.

3) The step size controlling parameter of the wall deformation, $\varepsilon$, is locally adjusted such that the distance between any of the two boundary nodes cannot be smaller than $r_{0}$ and no segment along the updated boundary intersects with each other. Also the $y$-coordinates of the nodes on $\Gamma_{\mathrm{M}}$ are kept larger than or equal to 0 , baseline of the fin surface (the boundary $\Gamma_{\mathrm{D}}$ ).

In the present bubble mesh method, most of the computational time is spent on the force calculation in Procedure (ii) above, which requires the search for the neighboring bubbles within a cutoff radius $\alpha_{B} r_{0}$. We reduce the computational time by introducing an adaptive resolution (AR) cell list (Awile et al., 2012). In the AR cell lists, an adaptive tree data structure is employed, and each node is assigned to one of the different tree levels that are divided according to the cutoff radius.

Figure 2 shows the computational time required for calculating the bubble-bubble interaction force versus the total number of nodes. Here we assume the same configuration as in a benchmark test in a two-dimensional rectangular region (Fig. 2a), combining large node-spacing zone (Zone I) and small node-spacing zone (Zone II) connected on the right to Zone I (Awile et al., 2012). The node spacing for each zone is constant at $h_{\mathrm{I}}$ and $h_{\mathrm{II}}$, respectively, and the size ratio is defined as $\lambda=h_{\mathrm{I}} / h_{\mathrm{II}}$. Each node has a constant bubble radius of $1.5 \times h_{\mathrm{I}}$ and $1.5 \times h_{\mathrm{II}}$ for Zones I and II, 
(a)

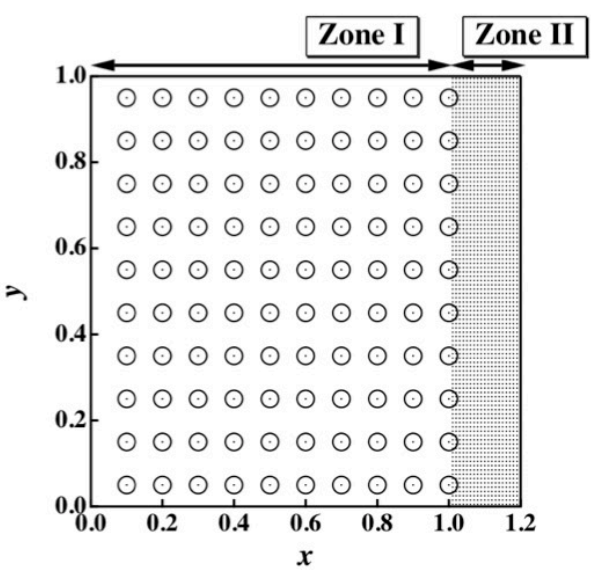

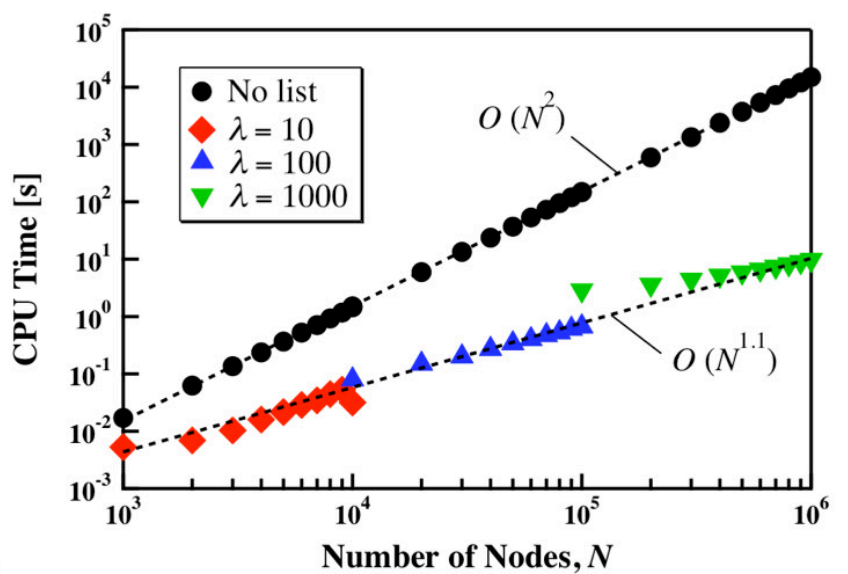

(b)

Fig. 2 Efficiency of the AR cell list: (a) configuration of a benchmark test, (b) computational time for the force calculation versus the number of bubbles with different size ratio, $\lambda$.

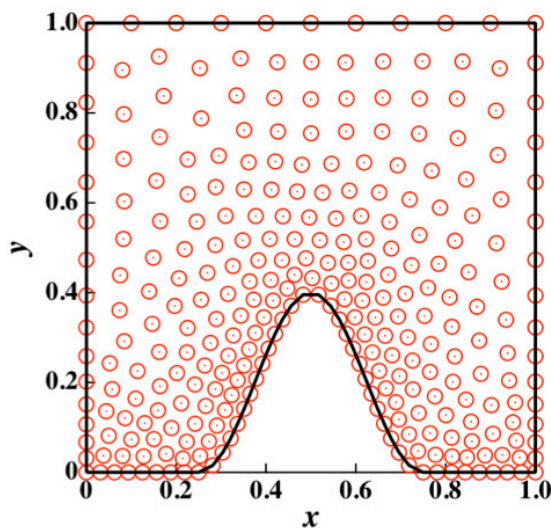

(a)

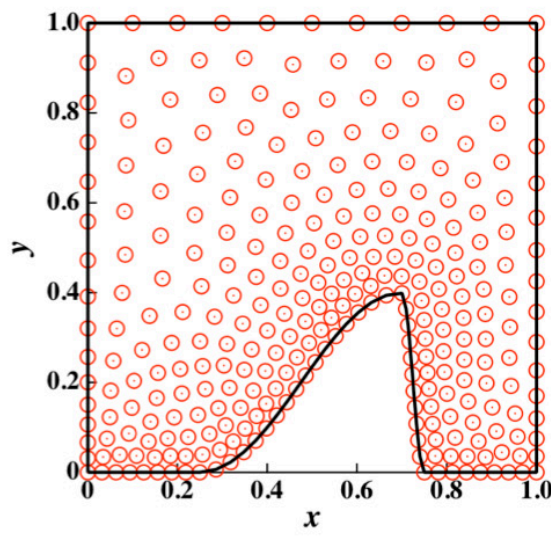

(b)

Fig. 3 Node distributions generated by the present bubble mesh method for different fin shapes determined by Eq. (14). The same density function, $d=\min (0.02+0.06 \times l, 0.05)$, is used: (a) $A=0.4, x_{c}=0.5$, (b) $A=0.4, x_{c}=0.7$.

respectively. The number of nodes in Zones I and II are 100 and $N$, respectively. In Fig. 2b, the CPU time for completing the force calculation with the AR cell lists for different expansion factors, i.e. $\lambda=10,100$, and 1000, are displayed. The force calculation result without cell lists is also plotted for comparison. It is shown that the computational time is much reduced to $O(N)$ with the present AR cell list, whereas it is $O\left(N^{2}\right)$ without the cell lists, showing that the AR cell list is effective even for large size ratios. Therefore, the AR cell list is used in the generation of bubble mesh in the present study.

Figure 3 shows the node distributions generated by the present bubble mesh method. For two different fin shapes, the same node density function is used, where $l$ denotes the minimum distance to the fin surface. According to the density function, the equilibrium node positions are obtained after the force relaxation and the packing-factor adjustment. The numbers of the total nodes generated are 293 and 294 for Figs. 3a and 3b, respectively. Note that the nodal points are exactly dotted in Fig. 3, while the actual radius of each bubble is not drawn exactly. In these examples, the radius of the bubble enlarges with the distance from the bottom boundary. It is seen that almost similar spatial resolution is obtained over the whole domain for different boundary shapes. It is also seen that, even for a symmetrical domain as shown in Fig. 3a, the node distribution generated exhibits some asymmetric features, which might affect the optimum shape obtained.

\section{Results of the shape optimization}

We evaluate the present shape-optimization scheme through a series of MLPG analyses. Different initial shapes of the fin geometry are considered. The initial surface profiles for the boundary $\Gamma_{M}$ are given by: 


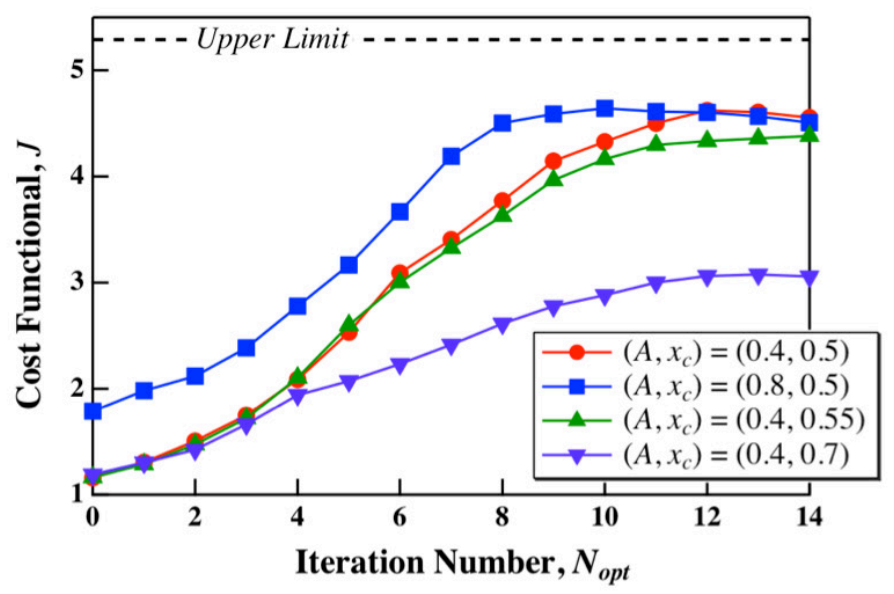

Fig. 4 Evolution of the cost functional $J$ for different initial shapes versus the iteration number $N_{\text {opt }}$.

(a)
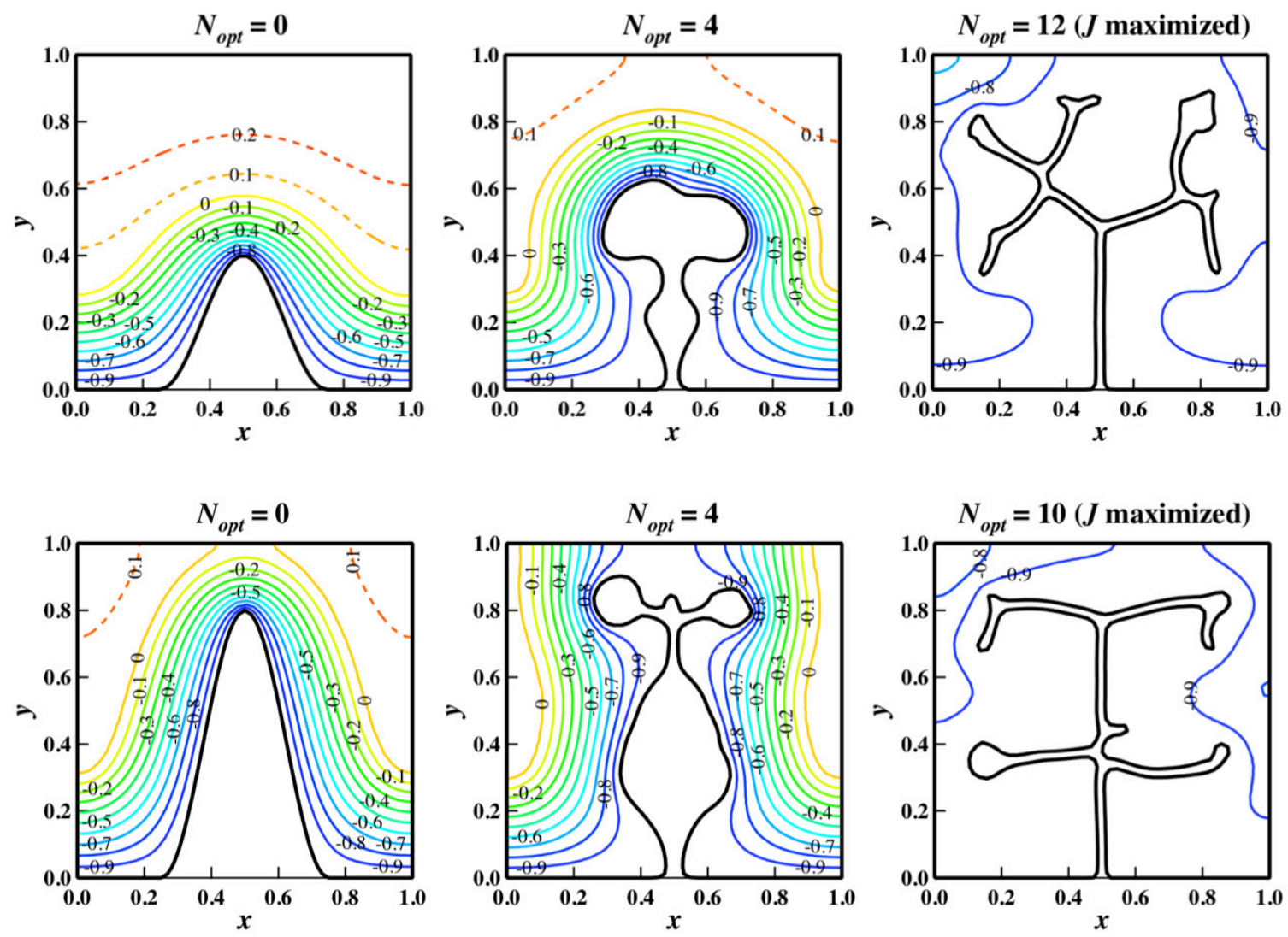

Fig. 5 Evolution of the fin shape and the iso-contours of the temperature at $t=t_{\max }$ for different initial wave amplitudes: (a) $\left(A, x_{c}\right)=(0.4,0.5)$, (b) $\left(A, x_{c}\right)=(0.8,0.5)$.

$$
y_{w}=\left\{\begin{array}{l}
A \sin ^{2}\left(\frac{\pi}{2} \frac{x-0.25}{x_{c}-0.25}\right) \quad\left(0.25<x \leq x_{c}\right), \\
A \sin ^{2} \frac{\pi}{2}\left(\frac{x-x_{c}}{0.75-x_{c}}+1\right) \quad\left(x_{c}<x<0.75\right) .
\end{array}\right.
$$

Here, the sinusoidal wave is symmetric for $x_{c}=0.5$ and asymmetric for $x_{c} \neq 0.5$. The bottom boundary denoted as $\Gamma_{\mathrm{D}}$ in Fig. 1 is kept the same during the shape optimization. In the present computation, the following parameters are used: $k_{\mathrm{L}} / k_{\mathrm{S}}=1, c_{\mathrm{L}} / c_{\mathrm{S}}=1$, Ste $=1, T_{0}=0.3, \Delta t=0.001$, and $\varepsilon_{\mathrm{T}}=0.05$. For the node arrangement in the MLPG analyses, we 
(a)
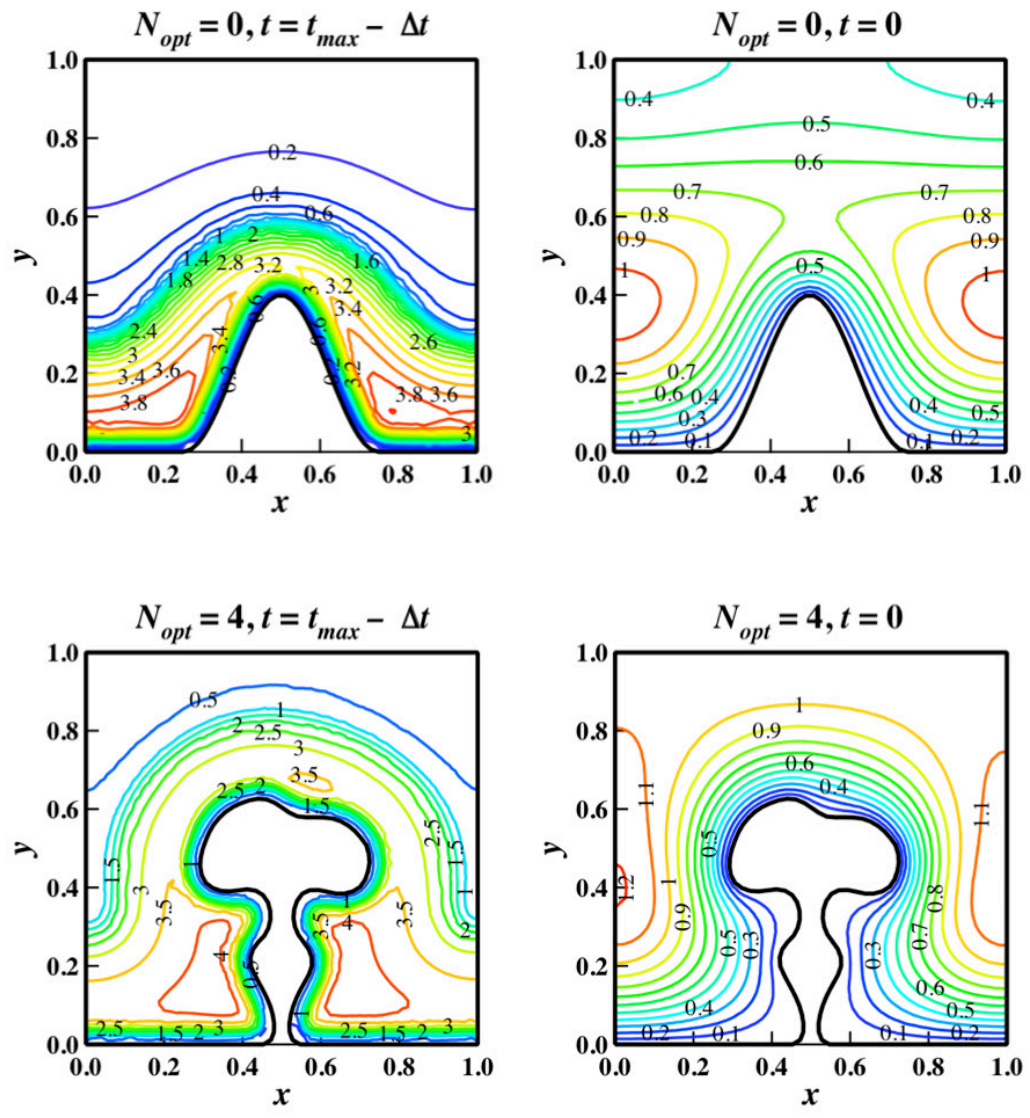

Fig. 6 Iso-contours of the adjoint enthalpy fields at $t=t_{\max }-\Delta t$ and $t=0$ for the initial shape with $\left(A, x_{c}\right)=(0.4,0.5)$ :

(a) $N_{\text {opt }}=0$, (b) $N_{\text {opt }}=4$.

employ a constant node-density function, given by $d=0.0125$. Thus, the node spacing is kept constant at $R \sim 0.025$. The number of nodes generated ranges from 1800 to 2050, reflecting the change of the fin geometry. For the shape modification, the value of $\varepsilon$ is initially set as $\varepsilon=0.01$. In this paper, optimization results for four sets of the initial fin shape, i.e. $\left(A, x_{c}\right)=(0.4,0.5),(0.8,0.5),(0.4,0.55)$, and $(0.4,0.7)$, are shown. The terminal time $t_{\max }$ used in the cost functional $J$ defined by Eq. (4) is set as $t_{\max }=0.05$ throughout the present computation.

Figure 4 shows the change of the cost function $J$ for different initial shapes versus the iteration number $N_{\text {opt }}$. The present $J$ defined by Eq. (4) is evaluated by a domain integral based on a background Cartesian square mesh having $100 \times 100$ nodes. The upper limit of $J$, where the liquid temperature equals to the wall temperature everywhere at $t=$ $t_{\max }$, is also plotted. It is shown that, regardless of the initial shape, the present cost function increases in a monotonic way with the iteration number to reach its maximum. It is also seen that the $J$ values for $\left(A, x_{c}\right)=(0.4,0.5),(0.8,0.5)$, and $(0.4,0.55)$, increase up to the same level except for $\left(A, x_{c}\right)=(0.4,0.7)$, where $J$ follows a different path.

Figure 5 shows the change of the fin shape during the optimization for different initial wave amplitudes. The iso-contours of the temperature at $t=t_{\max }$ are also displayed. At the initial shape, the phase change interface, corresponding to the contour line of $T=0$, lies in the middle of the fluid region. With increasing the iteration number, the fin shape is drastically changed; The width of the fin shrinks near its bottom region, and the rounded zones are divided into narrower branches. In the present shape evolution process, when $N_{\text {opt }}<4$, the fin shape is modified with a constant step size parameter of $\varepsilon=0.01$ all along $\Gamma_{\mathrm{M}}$. After $N_{o p t}=4$, the elongated tree-like structures are generated, and the node rearrangement is restricted by locally downsizing the value of $\varepsilon$ such that the minimum width is larger than the minimum resolution $(2 R)$ of the present analysis. Without this treatment, two sides of the fin intersect with each other and the computation will not proceed. In both cases, the maximum fin height of $\sim 0.8$ is obtained, and the lateral protrusion distance of left and right arms is $\sim 0.4$. It is shown that, despite the topological difference of the optimized shapes for $A=0.4$ and 0.8 , the corresponding temperature fields exhibit a global similarity. Therefore, heat transfer enhancement over the whole domain can be achieved with the present shape optimization scheme. 


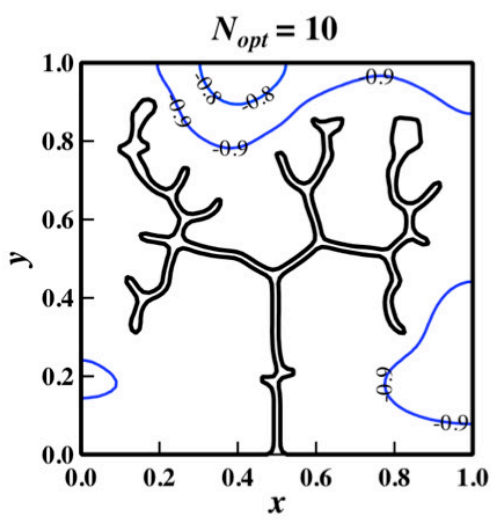

Fig. 7 Optimized shape with a higher node density of $d=0.0083$ for the initial shape with $\left(A, x_{c}\right)=(0.4,0.5)$.

(a)
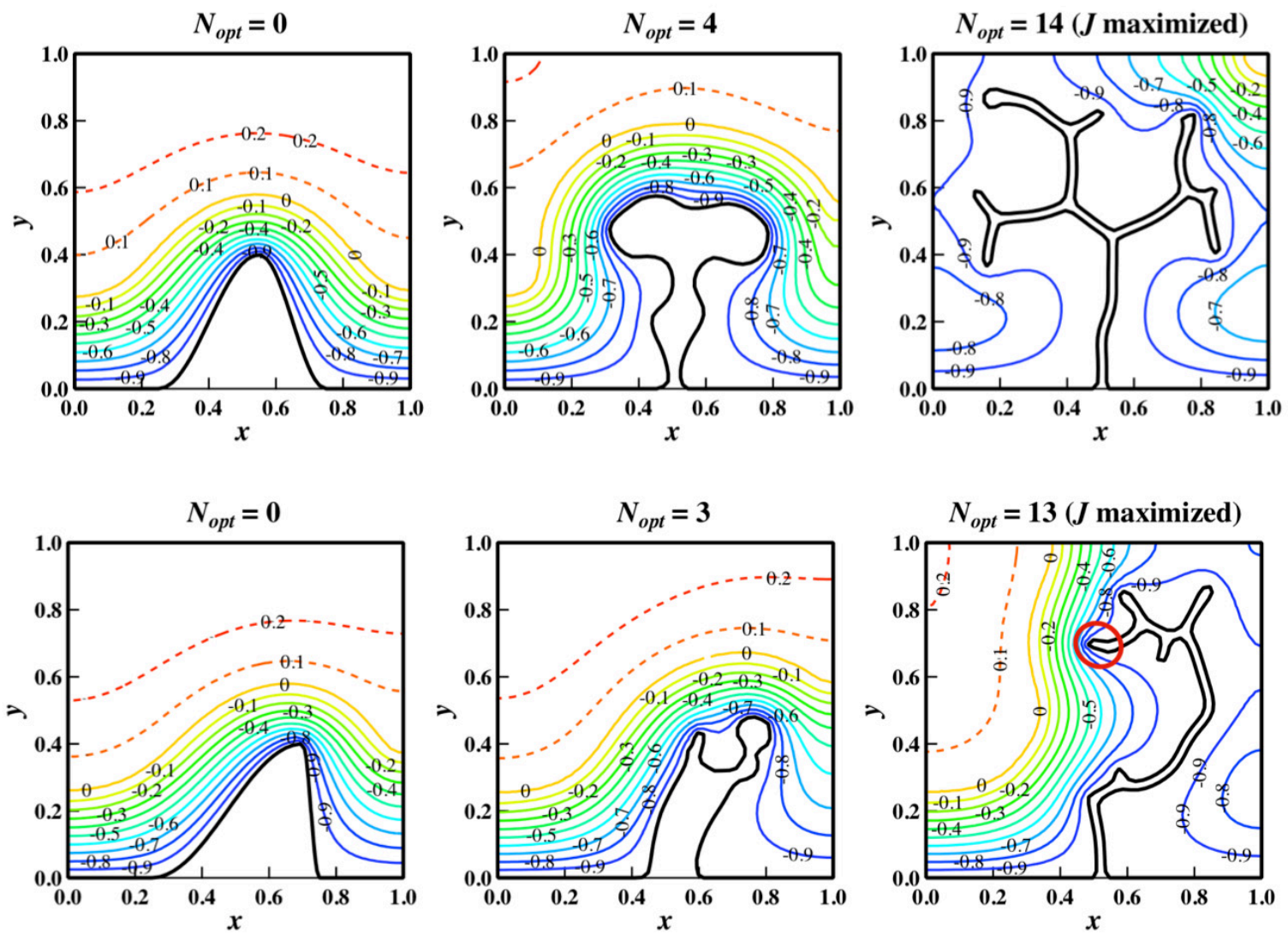

Fig. 8 Evolution of the fin shape and the iso-contours of the temperature at $t=t_{\max }$ for different asymmetry of the initial shape: (a) $\left(A, x_{c}\right)=(0.4,0.55)$, (b) $\left(A, x_{c}\right)=(0.4,0.7)$.

It is noted here that the present computational nodes are allocated using the bubble-mesh method for the boundary shape to be modified. The node distribution is asymmetric due to the relaxation process of the local bubble-bubble interaction force. Due to this asymmetric node allocation, the fin shape, even if it is initially symmetric, loses its symmetry as the iteration proceeds. However, the numerical accuracy of the temperature and adjoint enthalpy fields should be kept by using the boundary-fitted node allocation with the present bubble-mesh method. Since the optimized temperature field has shown global symmetry and similarity for different initial shapes, the topological asymmetry itself is considered to have minor impact on the maximized value of the present cost function.

Figure 6 shows the iso-contours of the adjoint enthalpy fields for the initial shape of $\left(A, x_{c}\right)=(0.4,0.5)$. The adjoint enthalpy fields at $t=\left(t_{\max }-\Delta t\right)$ and $t=0$ for the iteration number of $N_{\text {opt }}=0$ (initial state) and 4 are shown. Due to the 
initial condition given by Eq. (10), the adjoint enthalpy has its peak near the bottom region of the fin at $t \sim t_{\max }$. Since the adjoint enthalpy is decreased through the adjoint heat flux to the fin surface (constant $H^{*}$ boundary as set in Eq. (10)), the peak zone diffuses into the fluid domain as time progresses inversely to $t=0$. The shape deformation derived in Eq. (12) is determined based on the product of the gradients of the temperature and the adjoint enthalpy fields, and thus the amount of the deformation is intensified locally near the tip of the fin.

Thinned tree-like structure obtained in the present optimization is reasonable, since the penalty for extending the heat transfer surface area such as the temperature drop along the fin is not considered in the formulation of the adjoint analysis. The shape obtained should depend on the number of nodes, so that the present optimization problem is not well posed (Allaire et al., 2004), i.e., no unique solution exists.

Figure 7 shows an additional computational result with a higher node density $(d=0.0083,1.5$ times denser than for Fig. 5a). It is seen that even thinner structures are generated for a higher node density, corresponding to the reduced minimum spacing of the computational nodes. On the other hand, the maximum value of the cost function with $d=$ 0.0083 is 4.724 , which is only $2 \%$ higher than that for $d=0.0125$. Since the optimized temperature fields again exhibit global similarity despite small topological discrepancies, the spatial resolution with $d=0.0125$ is enough for proving the effectiveness of the present shape-optimization approach.

If a constraint for thinned structure is imposed, the ill-posedness is expected to be regularized. One possibility is the perimeter constraint (Ambrosio \& Buttazzo, 1993). From an application point of view, the fin-efficiency effect, caused by the heat conduction inside the fin solid material (Morimoto \& Suzuki, 2016), gives another penalty for the high-aspect-ratio extended heat transfer surfaces.

Figure 8 shows the change of the fin shape with different values of $x_{c}$ for the initial shape. For the case of weak asymmetry with $x_{c}=0.55$, the shape evolution progresses in a similar way as for the symmetrical case with $x_{c}=0.5$ (Fig. 8a). On the other hand, for the case of strong asymmetry with $x_{c}=0.7$, the fin shape follows a different evolution path (Fig. 8b), which falls in a smaller value of $J$ as shown previously in Fig. 4. For the latter case, since the amount of the fluid heat capacity on the right side of the fin becomes significantly smaller than that on the left side, the steeper gradients of the temperature and the adjoint enthalpy fields are induced on the right side. Thus, the fin upward growth is tilted in the positive $x$-direction. Although the thinned structure cannot be modified further due to the numerical constraints around the tip of the fin (circled in Fig. 8b, the right), the shape deformation function expressed in Eq. (12) still looks for further elongation toward the left-side fluid domain. As such, the present shape optimization scheme provides a suggestive direction for further increase of $J$, even for locally trapped state. It is indicated that the present shape optimization method is rather robust for a wide range of the initial guess to find the optimal solution.

\section{Conclusion}

We have formulated an adjoint-based shape-optimization scheme in liquid/solid phase change heat transfer problem under the isothermal wall temperature condition. We derived the surface deformation rule based on the variational information of the cost functional, which is calculated from the physical temperature and the adjoint enthalpy fields. For the computation of the developed scheme, we have implemented a meshless local Petrov-Galerkin (MLPG) method, and the effectiveness of the present shape-optimization scheme has been verified. It is shown that the boundary-fitted node arrangement using the bubble mesh allows for high-resolution computation with complex boundary geometry. In the present study, we have focused on the effect of the initial geometry upon the shape evolution process. It is demonstrated that, within a certain range of the initial state, the present shape-optimization scheme successively modifies the fin shape to generate tree-like structure. Despite the topological differences of the final shapes, similar temperature fields are obtained for enhancement of the solidification process.

\section{References}

Alifanov, O. M., Solution of an inverse problem of heat conduction by iteration methods, Journal of Engineering Physics, Vol. 26, No. 4 (1974), pp. 471-476.

Allaire, G., Jouve, F., and Toader, A.-M., Structural optimization using sensitivity analysis and a level-set method, Journal of Computational Physics, Vol. 194, No. 1 (2004), pp. 363-393.

Ambrosio, L., Buttazzo, G., An optimal design problem with perimeter penalization, Calculus of Variations and Partial Differential Equations, Vol. 1, No. 1 (1993), pp. 55-69. 
Atluri, S. N., and Zhu, T., A new meshless local Petrov-Galerkin (MLPG) approach in computational mechanics, Computational Mechanics, Vol. 22, No. 2 (1998), pp. 117-127.

Awile, O., Büyükkeçeci, F., Reboux, S., and Sbalzarini, I. F., Fast neighbor lists for adaptive-resolution particle simulations, Computer Physics Communication, Vol. 183, No. 5 (2012), pp. 1073-1081.

Bendsøe, M. P., and Sigmund, O., Topology Optimization - Theory, Methods, and Applications, Springer, Berlin Heidelberg, New York (2004).

Bewley, T. R., Moin, P., and Temam, R., DNS-based predictive control of turbulence: an optimal benchmark for feedback algorithms, Journal of Fluid Mechanics, Vol. 447 (2001), pp. 179-225.

Çabuk, H., and Modi, V., Optimum plane diffusers in laminar flow, Journal of Fluid Mechanics, Vol. 237 (1992), pp. 373-393.

Deng, Y., Liu, Z., Zhang, P., Liu, Y., and Wu, Y., Topology optimization of unsteady incompressible Navier-Stokes flows, Journal of Computational Physics, Vol. 230, No. 17 (2011), pp. 6688-6708.

Gersborg-Hansen, A., Bendsøe, M. P., and Sigmund, O., Topology optimization of heat conduction problems using the finite volume method, Structural and Multidisciplinary Optimization, Vol. 31, No. 4 (2006), pp. 251-259.

Goldberg, D. E., Genetic Algorithms in Search, Optimization, and Machine Learning, Addison-Wesley, Reading, MA (1989).

Iga, A., Nishiwaki, S., Izui, K., and Yoshimura, M., Topology optimization for thermal conductors considering design-dependent effects, including heat conduction and convection, International Journal of Heat and Mass Transfer, Vol. 52, No. 11 (2009), pp. 2721-2732.

Jameson, A., Aerodynamic design via control theory, Journal of Scientific Computing, Vol. 3, No. 3 (1988), pp. 233-260.

Kandasamy, R., Wang, X.-Q., and Mujumdar, A. S., Transient cooling of electronics using phase change material (PCM)-based heat sinks, Applied Thermal Engineering, Vol. 28, No. 8-9 (2008), pp. 1047-1057.

Kasagi, N., Hasegawa, Y., Fukagata, K., and Iwamoto, K., Control of turbulent transport: Less friction and more heat transfer, Transactions of the ASME, Journal of Heat Transfer, Vol. 134, No. 3 (2012), 031009 (10 pp.).

Lewis, R. W., and Roberts, P. M., Finite element simulation of solidification problems, Applied Scientific Research, Vol. 44, No.1 (1987), pp. 61-92.

Lim, S., and Choi, H., Optimal shape design of a two-dimensional asymmetric diffuser in turbulent flow, AIAA Journal, Vol. 42, No. 6 (2004), pp. 1154-1169.

Lions, J., Optimal Control of Systems Governed by Partial Differential Equations (1971), Springer, New York.

Liu, G. R., and Gu, Y. T., An Introduction to Meshfree Methods and Their Programming, Springer (2005).

Loulou, T., and Scott, E. P., An inverse heat conduction problem with heat flux measurements, International Journal for Numerical Methods in Engineering, Vol. 67, No. 11 (2006), pp. 1587-1616.

Mittal, R., and Iaccarino, G., Immersed boundary methods, Annual Review of Fluid Mechanics, Vol. 37 (2005), pp. 239-261.

Mohammadi, B., and Pironneau, O., Shape optimization in fluid mechanics, Annual Review of Fluid Mechanics, Vol. 36 (2004), pp. 255-279.

Molho, J. I., Herr, A. E., Mosier, B. P., Santiago, J. G., Kenny, T. W., Brennen, R. A., Gordon, G. B., and Mohammadi, B., Optimization of turn geometries for microchip electrophoresis, Analytical Chemistry, Vol. 73, No. 6 (2001), pp. 1350-1360.

Morimoto, K., Suzuki, Y., and Kasagi, N., Optimal shape design of compact heat exchangers based on adjoint analysis of momentum and heat transfer, Journal of Thermal Science and Technology, Vol. 5, No. 1 (2010), pp. 24-35.

Morimoto, K., and Suzuki, Y., Adjoint-based optimum thermal control of laser diodes, Proc. 15th International Heat Transfer Conference, IHTC15-9670 (10 pp.) (2014).

Morimoto, K., and Suzuki, Y., Adjoint-based shape optimization of extended heat transfer surfaces for enhanced solidification process with heat conduction in the solid wall, Proc. 1st Pacific Rim Thermal Engineering Conference, PRTEC-15161 (5 pp.) (2016).

Papoutsis-Kiachagias, E. M., and Giannakoglou, K. C., Continuous adjoint methods for turbulent flows, applied to shape and topology optimization: Industrial applications, Archives of Computational Methods in Engineering, Vol. 23, No. 2 (2016), pp. 255-299.

Peskin, C. S., Numerical analysis of blood flow in the heart, Journal of Computational Physics, Vol. 25, No. 3 (1977), 
pp. 220-252.

Pironneau, O., Optimum profiles in Stokes flow, Journal of Fluid Mechanics, Vol. 59 (1973), pp. 117-128.

Pironneau, O., Optimal Shape Design for Elliptic Systems, Springer-Verlag, New York (1984).

Reuther, J., Jameson, A., Farmer, J., Martinelli, L., and Saunders, D., Aerodynamic shape optimization of complex aircraft configurations via an adjoint formulation, AIAA Paper 96-0094 (1996).

Reynolds, D., McConnachie, J., Bettess, P., Christie, W. C., and Bull, J. W., Reverse adaptivity - a new evolutionary tool for structural optimization, International Journal for Numerical Methods in Engineering, Vol. 45, No. 5 (1999), pp. 529-552.

Sethian, J. A., Level-Set Methods and Fast Marching Methods: Evolving Interfaces in Computational Geometry, Fluid Mechanics, Computer Vision and Materials Science, Cambridge University Press, Cambridge, MA (1999).

Sethian, J. A., and Wiegmann, A., Structural boundary design via level-set and immersed interface methods, Journal of Computational Physics, Vol. 163, No. 2 (2000), pp. 489-528.

Sharma, A., Tyagi, V. V., Chen, C. R., and Buddhi, D., Review on thermal energy storage with phase change materials and applications, Renewable and Sustainable Energy Reviews, Vol. 13, No. 2 (2009), pp. 318-345.

Shimada, K., and Gossard, D. C., Automatic triangular mesh generation of trimmed parametric surfaces for finite element analysis, Computer Aided Geometric Design, Vol. 15, No. 3 (1998), pp. 199-222.

Subaşi, M., Optimal control of heat source in a heat conductivity problem, Optimization Methods and Software, Vol. 17, No. 2 (2002), pp. 239-250.

Swaminathan, C. R., and Voller, V. R., On the enthalpy method, International Journal of Numerical Methods for Heat and Fluid Flow, Vol. 3, No. 3 (1993), pp. 233-244.

Thakur, H., Singh, K. M., and Sahoo, P. K., Phase change problems using the MLPG method, Numerical Heat Transfer Part A: Applications, Vol. 59, No. 6 (2011), pp. 438-458.

Voller, V. R., An overview of numerical methods for phase change problems, Advances in Numerical Heat Transfer, Vol. 1, pp. 341-375 (1996).

Walther, B., and Nadarajah, S., Constrained adjoint-based aerodynamic shape optimization of a single-stage transonic compressor, Transaction of the ASME, Journal of Turbomachinery, Vol. 135, No. 2 (2012), 021017 (10 pp.).

Zabaras, N., and Kang, S., On the solution of an ill-posed design solidification problem using minimization techniques in finite- and infinite-dimensional function spaces, International Journal for Numerical Methods in Engineering, Vol. 36, No. 23 (1993), pp. 3973-3990.

Zhou, S., and Li, Q., A variational level set method for the topology optimization of steady-state Navier-Stokes flow, Journal of Computational Physics, Vol. 227, No. 24 (2008), pp. 10178-10195.

Zhuang, C. G., Xiong, Z. H., and Ding, H., A level set method for topology optimization of heat conduction problem under multiple load cases, Computer Methods in Applied Mechanics and Engineering, Vol. 196, No. 4-6 (2007), pp. 1074-1084.

Zienkiewicz, O. C., Taylor, R. L., and Zhu, J. Z., The Finite Element Method: Its Basis and Fundamentals (6th edn.), Elsevier Butterworth-Heinemann (2005).

Zymaris, A. S., Papadimitriou, D. I., Giannakoglou, K. C., and Othmer, C., Adjoint wall functions: A new concept for use in aerodynamic shape optimization, Journal of Computational Physics, Vol. 229, No. 13 (2010), pp. 5228-5245. 